encephalopathy (HE) and infections respectively; 38.5\% died at 90-days follow-up. Specific bacteria were found to be associated with HE [Bifidobacteriaceae,Coriobacteriaceae], sepsis [Veillonellaceae,Prevotellaceae], CTP $>10$ [Bifidobacteriaceae, Synergistaceae], MELD $>25$ [Dehalobacteriaceae, Turicibacteraceae] and death [Enterobacteriaceae,Peptococcaceae]. Significantly higher relative abundance(RA) of Lachnobacterium, Catenibacterium associated with $\mathrm{HE}$ at-admission while orallyrepresented bacteria were associated with infections at admission. Propionibacterium, Fusobacteria were associated with DF $\geq 65$ while Eubacterium, Capnocytophaga were associated with CTP. Enhydrobacter and Pediococcus were preferentially abundant post-steroid-therapy. Aerococcus was associated with post-treatment death. Prevotella was associated with survival post steroid. Upregulation of phenylpropanoid-biosynthesis (innate-immunity) in those without follow-up infections and glycerophospholipid-metabolism(cellular-integrity) in those who died were significant. Co-occurrence between Christensenella, Prevotella and mutual-exclusion between Megamonas, Citrobacter was associated with $\mathrm{HE}$ at admission. Mutual-exclusion between Coprococcus eutactus, Catenibacterium and Megamonas was associated with infections at admission while Enterococcus cecorum, Acinetobacter schindleri, Mitsuokella were associated with AKI at admission (figure 1).

Conclusions Specific gut-microbiota, their interactions and metabolites are associated with complications of SAH as well as outcomes with steroid-therapy. Advanced metagenomicsbased precision-medicine as add-on treatments may be a novel therapeutic area for improving disease outcomes.

\section{IDDF2020-ABS-0151 FACTORS PREDICTING IN-HOSPITAL MORTALITY IN PATIENTS HOSPITALIZED FOR LIVER CIRRHOSIS: A FIVE YEARS RETROSPECTIVE REVIEW IN CAMEROON}

${ }^{1}$ Chi Fru McWright* ${ }^{*}{ }^{1}$ Anutebeh Ephiseans, ${ }^{1}$ Ewane Edwin Nkwelle, ${ }^{1}$ Bokalli Franciose Adrien, ${ }^{2}$ Teuwafeu Denis Georges, ${ }^{3}$ Luma Henry Namme. ${ }^{1}$ Faculty of Health Sciences, University of Buea, Cameroon; ${ }^{2}$ Buea Regional Hospital, Cameroon; ${ }^{3}$ Douala General Hospital, Cameroon

\subsection{6/gutjnl-2020-IDDF.35}

Background The global prevalence of liver cirrhosis has been on the rise, estimated at $4.5-9 \%$ from autopsy studies, projected at 50 million people affected in 2020 and ranked the 12 th leading cause of death. However, data on studies evaluating the outcome of patients hospitalized for liver cirrhosis in SSA is scarce. We aimed to investigate the clinical and laboratory factors which predict in-hospital mortality in patients admitted for cirrhosis.

Methods This was a five years retrospective review of 183 cases hospitalized for liver cirrhosis between 1st January 2014 to 31st December 2018 in the medical units of two referral hospitals in Cameroon (figure1). Independent variables investigated included: liver disease severity; clinical complications with severities; biomarker trends (using repeated measurements during hospitalization to compute the average, peak and minimum values for each patient) for serum sodium, potassium, creatinine, C-reactive protein, Neutrophil-to-Leukocyte ratio/ NLR and Absolute Neutrophil Counts/ANC). Outcome investigated was in-hospital mortality, SPSS version 25.0 used to analyse data, logistic regression model to determine predictors of in-hospital mortality and significance set at $\mathrm{P}<0.05$.

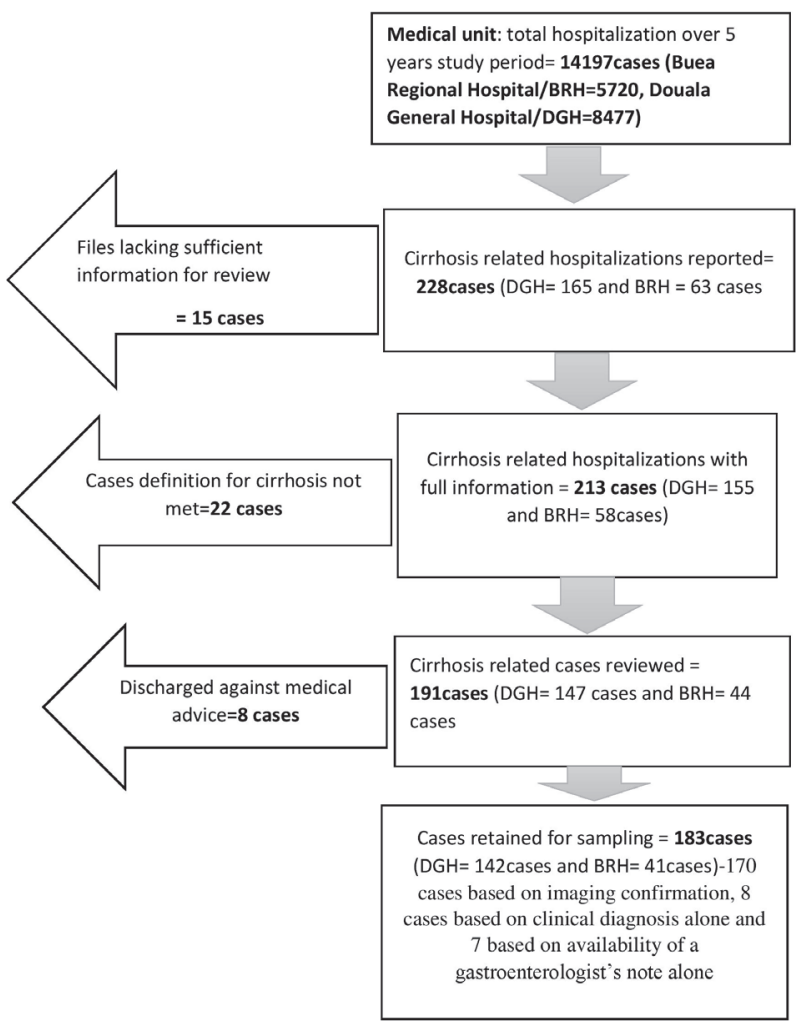

Abstract IDDF2020-ABS-0151 Figure 1 Flow chart demonstrating selection of sample population

Results Cirrhosis accounted for 16 cases per 1000 hospitalizations, with a male-to-female ratio of $1: 2$ and a mean age of $53( \pm 18)$ years. Most cases were hospitalized for acute decompensation $(93.4 \%)$ often presenting with ascites $(76.5 \%)$ and hepatic encephalopathy/HE (41.5\%). In-hospital mortality was $35.6 \%$, peaked within the first five days, with factors associated including: $\mathrm{HE}$ (OR, 95\% $\mathrm{CI}$ : 3.0, 1.6-5.6 $\mathrm{P}=$ $0.001)$, high Western-Haven grade $(\mathrm{P}=0.009)$, hyperkalemia (OR, 95\%CI: 3.7， 2.7-4.7 $\mathrm{P}=0.011$ ), elevated creatinine (OR,95\%CI: 3.0,2.3-3.8 P=0.004) and elevated absolute neutrophil count (OR,95\%CI: 2.6, 1.8-3.5 $\mathrm{P}=0.016)$. Following adjustments, Hyperkalaemia $(\mathrm{OR}=10, \mathrm{P}=0.003)$ and elevated absolute neutrophil count $(\mathrm{OR}=3.3, \mathrm{P}=0.047)$ were the independent predictors.

Conclusions In-Hospital mortality is very high in cirrhosis; it depends on a combination of factors and is predicted independently by hyperkalaemia and elevated absolute neutrophil count. Thus physicians should frequently reassess their clinical, inflammatory and metabolic status.

\section{IDDF2020-ABS-0178 SWITCHING OFF IMMUNOSUPPRESSIVE MYELOID CELLS BY TARGETING CELL CYCLE-RELATED KINASE PATHWAY: A NEW STRATEGY FOR COMBINATION IMMUNOTHERAPY}

Jingying Zhou*, Joseph Jao-yiu Sung, Alfred Sze Lok Cheng. The Chinese University of Hong Kong, Hong Kong

\subsection{6/gutjnl-2020-IDDF.36}

Background The immune-checkpoint-blockade (ICB) therapy has produced promising and yet modest objective response 